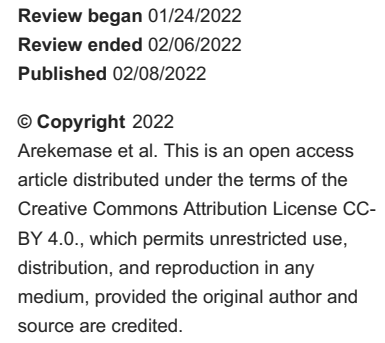

\section{Metaplastic Breast Carcinoma Presenting as a Mixed Solid and Cystic Lesion}

\author{
Hassan Arekemase ${ }^{1}$, Osmani Mohammed ${ }^{2}$, Usma Zafar $^{3}$, Kohli Manpreet ${ }^{4}$, Amin Maghari ${ }^{5}$
}

1. Anatomical and Clinical Pathology, Saint Barnabas Medical Center, Livingston, USA 2. Radiology, Monmouth Medical Center, Long Branch, USA 3. Pathology and Laboratory Medicine, Saint Barnabas Medical Center, Livingston, USA 4. Surgery, Monmouth Medical Center, Long Branch, USA 5. Pathology, Community Medical Center, Toms River, USA

Corresponding author: Hassan Arekemase, ennyhas@gmail.com

\section{Abstract}

The objective of this paper is to report a rare presentation of metaplastic breast carcinoma (MBC) as a mixed solid/cystic mass and emphasize the possibility of having a different biopsy report and final diagnosis.

MBC is a rare type of breast cancer consisting of mesenchymal or mixed mesenchymal and epithelial components. It is believed to result from the conversion of glandular cells into non-glandular cells through various forms of mutations. It is common in females and more frequent in older women. MBC is a triplenegative breast cancer (progestin receptor, estrogen receptor, and human epidermal growth factor receptor 2) with a very poor prognosis when compared with other types of breast cancers.

This is a case of an 84-year-old woman presenting with rapidly growing mixed solid and cystic breast mass with a final diagnosis of MBC. The initial biopsy report was high-grade ductal carcinoma in situ. This is an unusual presentation of MBC.

Categories: Pathology, Radiology, Oncology

Keywords: breast cancer, general surgery and breast cancer, breast cancer biology, breast cancer pathology, breast and gynaecological pathology, metaplastic breast cancer

\section{Introduction}

Breast cancer is the second most common cause of cancer-related deaths affecting the female population in the United States $[1,2]$. Metaplastic breast carcinoma (MBC) is a very rare variant of breast cancer, accounting for about $1 \%$ of all breast cancers [3,4]. MBC is defined as a group of variable breast diseases with mesenchymal or mixed mesenchymal and epithelial components $[5,6]$. It is a very rare type of breast cancer with a poor prognosis $[7,8]$.

According to the World Health Organization (WHO) 2019 classification of MBC, a few variants were identified: squamous cell carcinoma, low-grade adenosquamous carcinoma, spindle cell carcinoma, fibromatosis-like carcinoma, MBC with heterologous mesenchymal differentiation, and mixed MBC $[3,9]$.

The presentation is variable but mostly presents as a well-circumscribed or irregular solid mass. Occasionally, calcifications are identified in $\mathrm{MBC}$ with heterologous mesenchymal differentiation variants [4].

\section{Case Presentation}

An elderly woman presented with fatigue, weight loss, and a $10-\mathrm{cm}$ palpable right breast mass without any significant lymphadenopathy. The mass was painless, firm, and slightly mobile. No nipple discharge and no skin discoloration were noted. She had a past medical history of hypertension, end-stage renal disease on dialysis, and hip replacement. She denied fever, headache, blurring of vision, palpitation, chest pain, cough, breathing difficulties, change in bowel habits, back pain, and urinary symptoms. She had no family history of cancer, was not an alcoholic, quit smoking 25 years ago, and had no illicit drug use. She is married in a monogamous setting with three children and good family support.

Mammography reveals large lobulated low-density soft tissue mass in the right breast with relatively welldefined margins, the largest in the subareolar region. Breast ultrasound showed heterogeneous lobulated mixed solid and cystic mass in the right breast at the 1 o'clock position, $5 \mathrm{~cm}$ from the nipple (Figure 1 ). 


\section{Cureus}

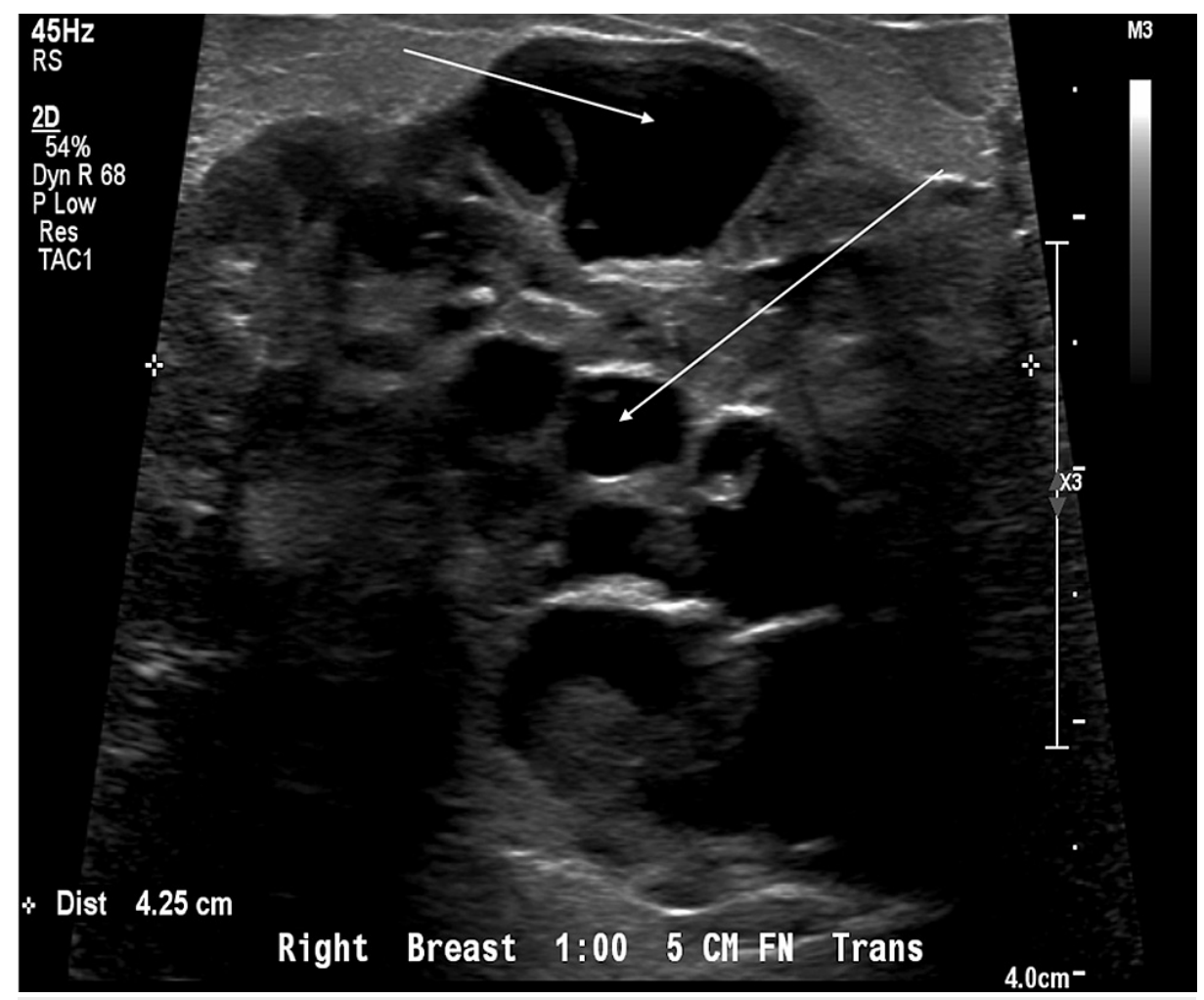

FIGURE 1: Ultrasound - transverse view.

Arrows pointing to the cystic spaces.

The biopsy was reported as multiple fragments of ductal carcinoma in situ, high grade with comedonecrosis. The resection specimen revealed a $10 \times 10 \mathrm{~cm}$ multilocular cystic and solid mass with necrosis and exudates (Figure 2). The light microscopy showed invasive carcinoma, high-grade carcinoma in situ, pleomorphism, mitosis, atypical spindle cell proliferation, and giant cells (Figures 3,4). The epithelial and spindle cell differentiation was positive for cytokeratin and OSCAR. Hormone receptor studies were all negative (Figure 5).

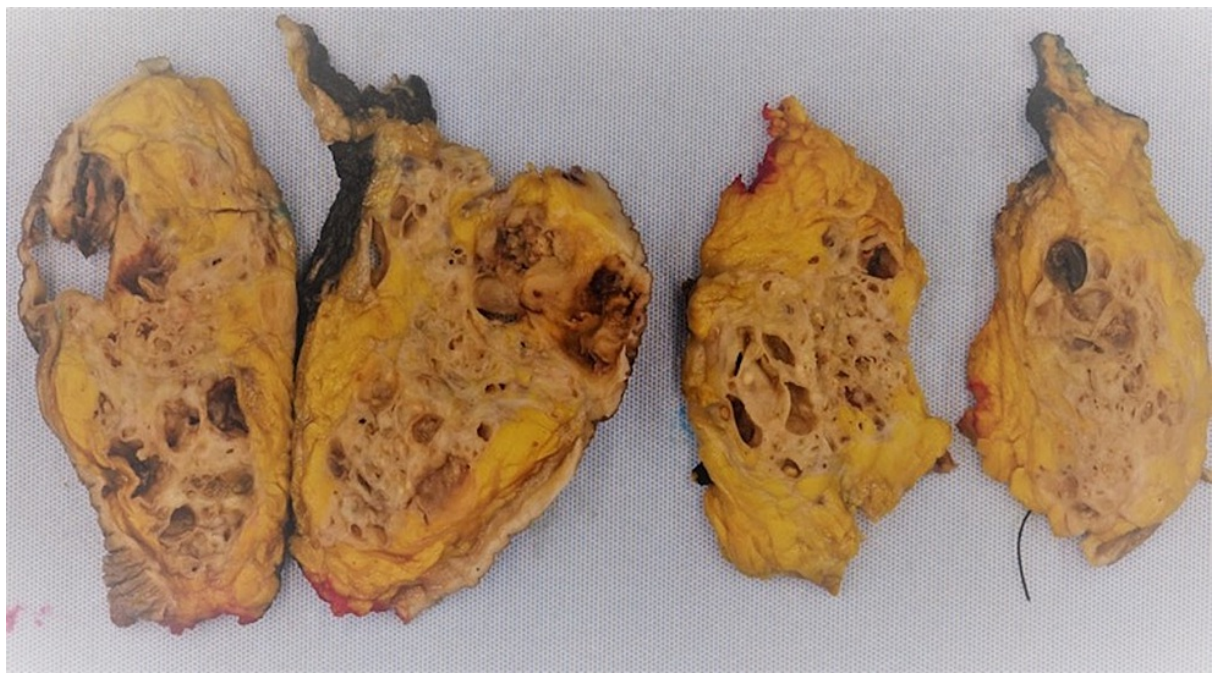

\section{FIGURE 2: Formalin-fixed breast resection.}

Gross appearance of brown-red tan solid and cystic lesion in a background of yellow tan fibrofatty breast tissue 


\section{Cureus}

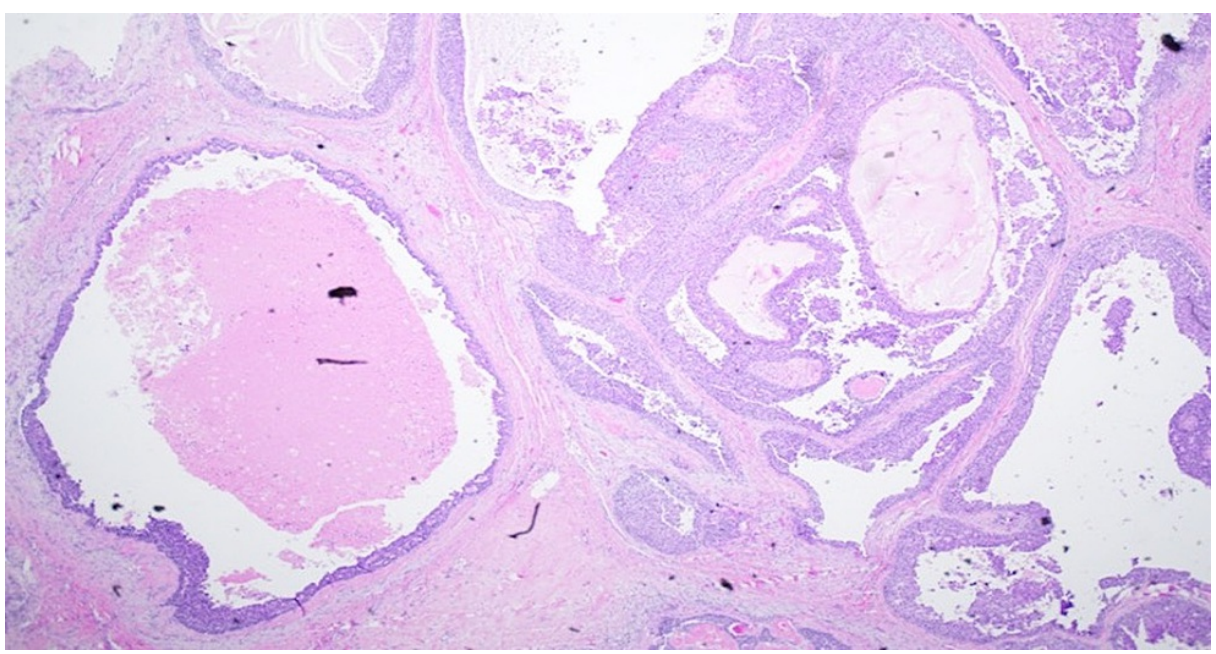

FIGURE 3: Low power image of solid and cystic areas.

Hematoxylin and eosin stain revealing cystic spaces with in situ, comedonecrosis, and invasive carcinoma.

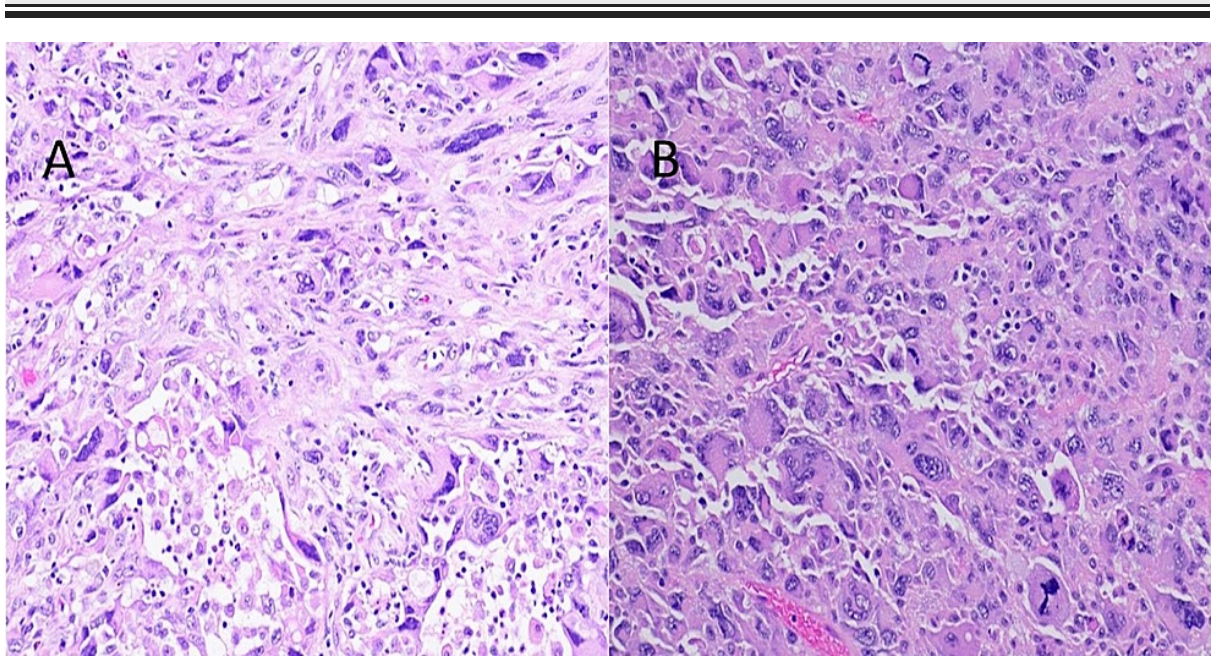

FIGURE 4: (A) Hematoxylin and eosin stain showing poorly differentiated tumor cells with pleomorphism, mitosis, and atypical spindle cell morphology. (B) Hematoxylin and eosin stain showing giant cells, pleomorphism, and mitosis. 


\section{Cureus}

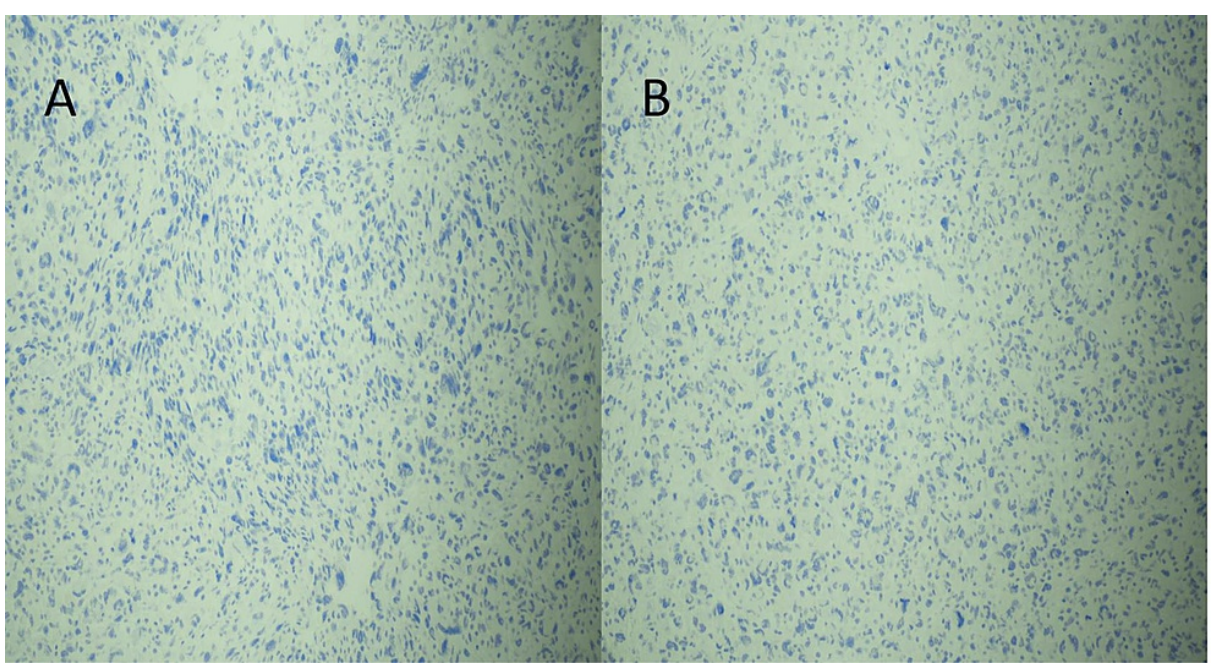

\section{FIGURE 5: Immunohistochemistry: (A) estrogen receptor and (B)}

progesterone receptor.

Immunohistochemical stain showing a negative estrogen and progesterone receptor.

\section{Discussion}

Metaplastic carcinoma of the breast was first described by Huvos in 1973 [10]. National Institute of Health classifies metastatic carcinoma of the breast as a rare disease [11]. The incidence is $0.2-5 \%$ of all breast cancer cases and the average age of presentation is 55 years [12]. Clinically, it presents as a wellcircumscribed palpable mass and is a well-delineated mass radiologically. The presentation is the same as invasive ductal carcinoma and not otherwise specified [12]. However, in our case, the lesion is a mixed cystic and solid. This is unusual and an important pattern to recognize.

The components of a metaplastic carcinoma can be epithelial, mesenchymal, or combined [13]. According to Huina Zhang, the epithelial-only carcinoma can be squamous cell carcinoma or adenosquamous carcinoma while the mesenchymal-only carcinoma can be fibromatosis-like metaplastic carcinoma and spindle cell carcinoma. Other elements present singly or in combination can be chondroid, osseous,

rhabdomyosarcomatous, angiosarcomatous, liposarcomatous, and neuroglial differentiation [14].

The spindle cell component may vary from spindle cell sarcoma to malignant fibrous histiocytoma-like sarcoma and can have syncytiotrophoblast-like giant cells [15]. Depending on the components present, the differential diagnosis can be quite variable including phyllodes tumor, primary breast sarcoma, myoepithelial carcinoma, metastatic sarcoma, adenomyoepithelioma, and pleomorphic adenoma. Wong et al. also suggested lobular carcinoma, pleomorphic carcinoma, and rare sarcomas such as angiosarcoma as differential [16].

The histological components in metaplastic carcinoma can be heterologous and immunohistochemistry is an essential tool in challenging cases. MBC is positive for basal cytokeratin including cytokeratin 5/6 (CK5/6) and 34BetaE12 [17]. According to Koker et al., p63 has a high sensitivity and specificity ( $86.7 \%$ and $99.4 \%$, respectively), and it may stain both the epithelial and spindle cell component. They suggested p63 may aid in the diagnosis of spindle cell tumors of the breast [18].

Richard et al. identified positivity of different markers helpful in the diagnosis of MBC: laminin 5 (96\%), p63 (57-86\%), CK5/6 (50-86\%), CD10 (85\%), smooth muscle actin (SMA) (60\%), and S-100 (45\%) [19]. The most important point to remember is that the presence of SMA and S-100 positivity does not rule out carcinoma. This is a fallout from the basic principle of breast pathology in which the presence of SMA, a myoepithelial marker, suggests a benign process. Han et al. have suggested the combination of OSCAR, cytokeratin 14 (CK14), and p63 is the most efficient panel (sensitivity 97.9\%) for diagnosing MBC [2].

MBC has a poorer prognosis as compared to other triple-negative breast carcinomas (TNBC). In a study by Song et al., the five-year overall survival rate is $54.5 \%$ (MPC) vs. $73.3 \%$ (TNBC). The study also concludes that the prognosis differs with the cellular component present in the MPC. Adenocarcinoma with spindle cell differentiation had the worst five-year overall survival rate at $40 \%$ [20].

\section{Conclusions}

For pathologists, extensive sampling of triple-negative breast lesions is important as there might be hidden 
metaplastic components which will impact the patient's survival rate and management. For radiologists, MBC can also present as a mixed solid and cystic lesion. A high index of suspicion and adequate biopsy sampling is important. For surgeons, even though the biopsy report read invasive or in situ carcinoma, resection specimen might reveal a different diagnosis due to extensive specimen sampling.

\section{Additional Information \\ Disclosures}

Human subjects: Consent was obtained or waived by all participants in this study. Conflicts of interest: In compliance with the ICMJE uniform disclosure form, all authors declare the following: Payment/services info: All authors have declared that no financial support was received from any organization for the submitted work. Financial relationships: All authors have declared that they have no financial relationships at present or within the previous three years with any organizations that might have an interest in the submitted work. Other relationships: All authors have declared that there are no other relationships or activities that could appear to have influenced the submitted work.

\section{Acknowledgements}

I would like to appreciate my attending for helping me learn pathology and identifying the important things to know.

\section{References}

1. Wu SG, Yang SP, Zhang WW, Wang J, Lian CL, Chen YX, He ZY: The longitudinal risk of mortality between invasive ductal carcinoma and metaplastic breast carcinoma. Sci Rep. 2020, 10:22070. 10.1038/s41598-02079166-5

2. Han M, Zhang H, Dabbs DJ: Best practice (efficient) immunohistologic panel for diagnosing metaplastic breast carcinoma. Appl Immunohistochem Mol Morphol. 2021, 29:265-9. 10.1097/PAI.0000000000000873

3. El Zein D, Hughes M, Kumar S, Peng X, Oyasiji T, Jabbour H, Khoury T: Metaplastic carcinoma of the breast is more aggressive than triple-negative breast cancer: a study from a single institution and review of literature. Clin Breast Cancer. 2017, 17:382-91. 10.1016/j.clbc.2017.04.009

4. Salemis NS: Metaplastic carcinoma of the breast with mesenchymal differentiation (carcinosarcoma). A unique presentation of an aggressive malignancy and literature review. Breast Dis. 2018, 37:169-75. 10.3233/BD-170313

5. Tekin L, Elibol FD, Bacaksızlar Sarı F, Nazlı O: Spindle cell metaplastic carcinoma of the breast . Breast J. 2020, 26:1421-2. 10.1111/tbj.13753

6. Alaoui M'hamdi H, Abbad F, Rais H, Asmouki H, Soumani A, Khouchani M, Belbaraka R: Rare variant of metaplastic carcinoma of the breast: a case report and review of the literature. J Med Case Rep. 2018, 12:43. 10.1186/s13256-017-1553-3

7. Yamashita M, Kamei Y, Murakami A, et al.: Metaplastic carcinoma of the breast and BRCA1 germline mutation: a case report and review. Hered Cancer Clin Pract. 2021, 19:3. 10.1186/s13053-020-00162-x

8. Takatsuka D, Ogura H, Asano Y, Nakamura A, Koizumi K, Shiiya N, Baba S: A difficult-to-diagnose fibromatosis-like metaplastic carcinoma of the breast: a case report. Surg Case Rep. 2021, 7:16. 10.1186/s40792-021-01110-0

9. Lei T, Pu T, Wei B, et al.: Clinicopathologic characteristics of HER2-positive metaplastic squamous cell carcinoma of the breast. J Clin Pathol. 2022, 75:18-23. 10.1136/jclinpath-2020-206468

10. Huvos AG, Lucas JC Jr, Foote FW Jr: Metaplastic breast carcinoma. Rare form of mammary cancer. N Y State J Med. 1973, 73:1078-82.

11. Metaplastic carcinoma of the breast. (2011). Accessed: January 1, 2022: https://rarediseases.info.nih.gov/diseases/10804/metaplastic-carcinoma-of-the-breast .

12. Reddy TP, Rosato RR, Li X, Moulder S, Piwnica-Worms H, Chang JC: A comprehensive overview of metaplastic breast cancer: clinical features and molecular aberrations. Breast Cancer Res. 2020, 22:121. 10.1186/s13058-020-01353-Z

13. Aydiner A, Sen F, Tambas M, et al.: Metaplastic breast carcinoma versus triple-negative breast cancer: survival and response to treatment. Medicine (Baltimore). 2015, 94:e2341. 10.1097/MD.0000000000002341

14. Breast, metaplastic carcinoma. (2021). Accessed: January 1, 2022: https://www.pathologyoutlines.com/topic/breastmalignantmetaplastic.html.

15. Altaf FJ, Mokhtar GA, Emam E, Bokhary RY, Mahfouz NB, Al Amoudi S, Al-Gaithy ZK: Metaplastic carcinoma of the breast: an immunohistochemical study. Diagn Pathol. 2014, 9:139. 10.1186/1746-1596-9139

16. Wong W, Brogi E, Reis-Filho JS, et al.: Poor response to neoadjuvant chemotherapy in metaplastic breast carcinoma. NPJ Breast Cancer. 2021, 7:96. 10.1038/s41523-021-00302-z

17. Hu J, Zhang H, Dong F, Zhang X, Wang S, Ming J, Huang T: Metaplastic breast cancer: treatment and prognosis by molecular subtype. Transl Oncol. 2021, 14:101054. 10.1016/j.tranon.2021.101054

18. Koker MM, Kleer CG: p63 expression in breast cancer: a highly sensitive and specific marker of metaplastic carcinoma. Am J Surg Pathol. 2004, 28:1506-12. 10.1097/01.pas.0000138183.97366.fd

19. Metaplastic carcinoma of the breast. (2009). Accessed: December 26, 2021: http://surgpathcriteria.stanford.edu/breast/metacabr/printable.html.

20. Song Y, Liu X, Zhang G, et al.: Unique clinicopathological features of metaplastic breast carcinoma compared with invasive ductal carcinoma and poor prognostic indicators. World J Surg Oncol. 2013, 11:129. 10.1186/1477-7819-11-129 